\title{
A Simulation-Based Framework for the Cooperation of VMS Travel Guidance and Traffic Signal Control
}

\author{
Meng Li, ${ }^{1}$ Han Jiang, ${ }^{2}$ Zhen Zhang, ${ }^{1}$ Wei Ni, ${ }^{1}$ Pinchao Zhang, ${ }^{1}$ and Jingyan Song ${ }^{2}$ \\ ${ }^{1}$ Department of Civil Engineering, Tsinghua University, 315 Shanheng He Building, Beijing 100084, China \\ ${ }^{2}$ Tsinghua National Laboratory for Information Science and Technology (TNList), Department of Automation, \\ Tsinghua University, Beijing 100084, China
}

Correspondence should be addressed to Han Jiang; jianghan0521@sina.cn

Received 14 January 2014; Revised 19 May 2014; Accepted 19 May 2014; Published 16 June 2014

Academic Editor: Wuhong Wang

Copyright (C) 2014 Meng Li et al. This is an open access article distributed under the Creative Commons Attribution License, which permits unrestricted use, distribution, and reproduction in any medium, provided the original work is properly cited.

\begin{abstract}
Nowadays, both travel guidance systems and traffic signal control systems are quite common for urban traffic management. In order to achieve collaborative effect, different models had been proposed in the last two decades. In recent years, with the development of variable message sign (VMS) technology, more and more VMS panels are installed on major arterials to provide highly visible and concise graphs or text messages to drivers, especially in developing countries. To discover drivers' responses to VMS, we establish a drivers' en route diversion model according to a stated-preference survey. Basically, we proposed a cooperative mechanism and systematic framework of VMS travel guidance and major arterials signal operations. And then a two-stage nested optimization problem is formulated. To solve this optimization problem, a simulation-based optimization method is adopted to optimize the cooperative strategies with TRANSIMS. The proposed method is applied to the real network of Tianjin City comprising of 30 nodes and 46 links. Simulations show that this new method could well improve the network condition by $26.3 \%$. And analysis reveals that GA with nested dynamic programming is an effective technique to solve the optimization problem.
\end{abstract}

\section{Introduction}

In recent years, China has entered the stage of quickened urbanization process. However, traffic problems such as traffic congestions and the deterioration of air quality are getting increasingly serious. Since the obvious unbalance between the rapidly growing traffic demand and the limitation of traffic resources, traffic management is becoming an increasingly serious concern. During the last two decades, how to alleviate traffic congestion by better urban traffic management strategies has captivated consistent attention from both traffic operators and engineers.

To reach this goal, various strategies have been developed for urban traffic management $[1,2]$. One important branch of such approaches is traffic flow guidance. Many researches have been conducted on traffic flow guidance by providing drivers with real-time information (e.g., traffic information broadcasting, on-board navigation systems, and variable message sign). Among them, the variable message sign installed on the roadside is designed to improve road network performance by providing such highly visible and concise information to drivers, especially congested conditions [3]. Instead of being installed on highways in America and European countries, VMS are developed to provide en route information for major arterials in developing countries. The application of VMS technology in congested urban road network in developing country can potentially ease traffic congestion. It was revealed that the key of effective utilization of VMS is to capture the relationship between variable messages and drivers' behavior [4]. Therefore, sound investigations of drivers' responses to VMS should be carried out at first.

Another common urban traffic management method is traffic signal operational strategy, which provides a realtime traffic flow control approach [5]. Obviously, there exist interactions and interdependence between travel guidance 
and traffic signal control. Consequently, analytical models or simulation evaluation seems inspiring by using traffic signal control system or travel guidance system separately. On the contrary, adopting traffic signal control and travel guidance simultaneously does not always succeed. Nowadays, more and more serious urban congestion problem in developing countries requires researchers' additional insights into the optimization of cooperative strategies.

In previous attempts, the cooperative mechanism related to traffic signal control and travel guidance is one of hotspots for researchers. In the early stage, most research integrates traffic signal control with user equilibrium [6-8] or dynamic user equilibrium (DUE) [9-11]. The key postulated in these studies is that all users have the same choice criterion and complete information. Taking this hypothesis, we find that this integration different from drivers' en route routechoice process neglects specific drivers' behaviors. More importantly, it is impossible to provide complete dynamic information for all drivers on urban road network. The researches on joint optimization of traffic signal control and UE or DUE cannot be put into practice so far.

To solve the aforementioned problems, some researches have been conducted on cooperation of en route travel guidance and traffic signal control. Among en route travel guidance approaches, advanced on-board navigation systems or traffic information broadcasting can provide real-time information and guidance advice for users. However, there are still some problems needed to be solved before using in developing countries: users of navigation systems only cover a tiny proportion of total travelers in developing countries; and it is isolated from signal control system. In general, these systems designed for travellers have to be separated from signal control systems for security reasons. Different with above approaches, the VMS strategically located on the roadside can directly affect drivers' en route choice. Moreover, VMS technique allows the information communication with road detectors and traffic signal controllers.

In recent years, several studies on VMS travel guidance and traffic signal control have been carried out for urban traffic management in developing countries $[12,13]$. Moreover, Lin further proposed a collaborative model integrating traffic control with VMS in a sudden disaster [14]. Nevertheless, these previous studies place emphasis on logistic steps and framework. In addition, only text messages (e.g., diversion advice and congestion information) are considered in previous attempts. In order to avoid the fluctuation of traffic flow, diversion advice should be published only if detour path owns obvious advantage. In other words, the diversion advice owns lag characteristic. To improve the effectiveness of travel guidance, we propose an active travel guidance method by a dynamic graphical display of the traffic status with colors in this paper. The application of VMS will definitely induce distributions of traffic flow in different paths. As a result, not considering the response of travellers may actually increase network-wide congestion. Therefore, we establish a valid driver diversion model on a behavioral survey before implementing the cooperative strategy.

In this paper, we first establish a logit model of drivers' responses to graph and text information on VMS panels based on a stated-preference survey. And then a cooperative strategy and systematical framework of VMS and traffic signal control are proposed for major arterials. Within this framework, the optimization of the cooperative strategy can be represented as a two-stage nested optimization problem. We name this optimization problem the VMS guidance and signal coordination (VGSC) problem. The desired road status display, diversion advice, and arterial signal parameters (i.e., cycle times and green times) are updated in the firststage optimization problem by using genetic algorithms. Subsequently, arterial signal offsets are optimized by dynamic programing method in the second-stage. Considering the complexity of interactions between vehicles and signalized or unsignalized intersections on a large network, we further develop a simulation-based optimization (SBO) software package with TRANSIMS to solve the two-stage nested optimization problem.

To explain the mechanism and application of the cooperative strategy, the rest of this paper is organized as follows. Section 2 builds the driver diversion model on the basis of a behavioral survey and propose the active travel guidance approach in detail. Section 3 proposes the cooperative strategy and establishes the two-stage nested optimization model. Section 4 presents the simulation-based optimization method to solve the optimization problem. Computational results on a particular network are presented in Section 5. Finally, Section 6 concludes our findings.

\section{Drivers' Diversion Model}

2.1. Stated-Preference Behavioral Survey. Many studies have investigated the impact of VMS on drivers' behavior [15, 16] and verified that the text message on VMS panels is a significant factor for route choice. The stated-preference (SP) survey is an effective tool to test the behavior of individuals. However, these SP surveys are not suitable for VMS installed in urban areas. Due to different scenarios including different message content, applied places, and traffic status, drivers' route choices would be quite different. Therefore, it is essential to carry out a sound behavioral survey for modeling drivers' behaviors.

In developing countries, VMS are widely used in urban road network. These VMS panels allow releasing information on "congestion scale," that is, road network with different colors. The "congestion scale" is a classification of average speed calculated by travel time (i.e., red means congested road segment; yellow means heavy traffic volume segment; and green means low-traffic segment). In detail, for major arterials, red represents the real-time speed lower than $20 \mathrm{~km} / \mathrm{h}$; yellow represents $20 \mathrm{~km} / \mathrm{h}-40 \mathrm{~km} / \mathrm{h}$; and green represents higher speed than $40 \mathrm{~km} / \mathrm{h}$. (see Figure 1) Previous research focuses on drivers' response to typical text messages while neglecting the graphical information on VMS panels.

To solve existing problems of drivers' responses to graphical information, we carried out a stated-preference (SP) survey, which is coded into pad computers. Considering road segments with different colors and detour distances that 


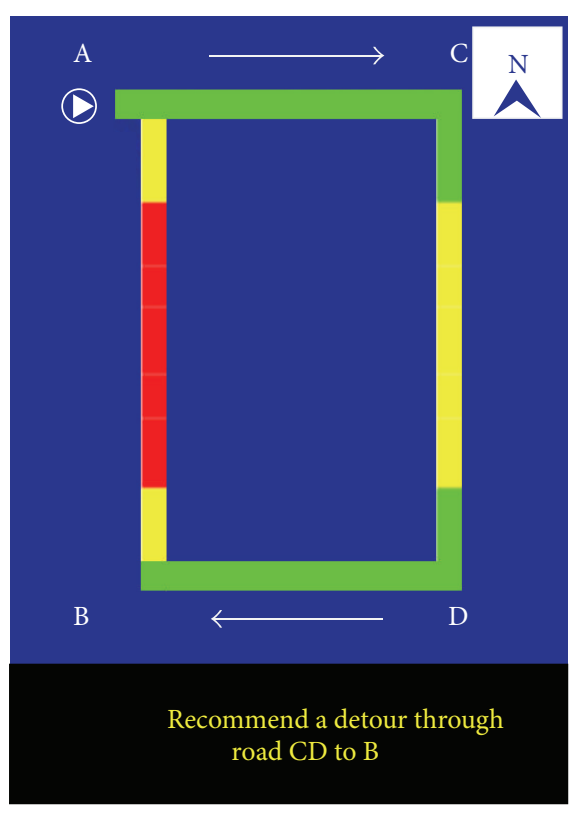

FIgURE 1: An illustration of VMS panel.

drivers might face in the urban area, various variables including distances of pretrip path $(\mathrm{AB})$ and detour route (ACDB), diversion advice, and colors were changed randomly.

Multiple factors vis-à-vis VMS have been considered in the SP survey and the collected information can be classified in the following categories:

(1) personal information: age, gender, and salary; and the personal attributes which define drivers' characteristics have been identified in Table 1;

(2) preference information:

(a) a drivers' acceptability of VMS;

(b) a drivers' response to information on VMS;

(3) dynamic information on VMS panels:

(a) distances: original route and alternative routes;

(b) colors: yellow and red segment ratios;

(c) diversion advice.

2.2. A Binary Logit Model of Drivers'Diversion Choice. On the basis of the SP survey, drivers' response to VMS is typically modeled by a binary logit model. There are two alternatives, which are denoted by 0 or 1 (i.e., 0 means keeping original route and 1 means choosing to divert). In order to recover all related factors, we take multiple variables into consideration, as follows:

(1) age: the drivers' ages;

(2) gender: a dummy variable, which is equal to zero if the driver is male and one otherwise;
TABLE 1: Summary of selected personal attributes.

\begin{tabular}{lcc}
\hline \multicolumn{2}{c}{ Personal attributes } & Percentage (\%) \\
\hline \multirow{2}{*}{ Gender } & Male & 44.94 \\
& Female & 55.06 \\
Age & $18-29$ & 39.33 \\
& $30-49$ & 58.43 \\
& $50-69$ & 2.25 \\
& $<50,000$ & 15.73 \\
Personal salary & $50,001-100,000$ & 21.35 \\
& $100,001-150,000$ & 20.22 \\
$150,001-200,000$ & 11.24 \\
$200,001-250,000$ & 16.85 \\
$250,001-300,000$ & 1.12 \\
& $300,001-350,000$ & 4.49 \\
$350,001-400,000$ & 1.12 \\
$>400,000$ & 7.87 \\
\hline
\end{tabular}

(3) route length: the length of routes $A B$ and $A C B$, denoted as $l_{\mathrm{AB}}$ and $l_{\mathrm{ACB}}$;

(4) advice: a dummy variable which is equal to zero if the VMS shows the advice and one otherwise;

(5) $\mathrm{YLR}_{\mathrm{AB} / \mathrm{ACB}}$ : yellow link ratio in routes $\mathrm{AB}$ and $\mathrm{ACB}$;

(6) $R L R_{A B / A C B}$ : red ratio in routes $A B$ and $A C B$. And, we have

$$
\operatorname{RLR}=\frac{L_{r}}{L_{y}+L_{g}+L_{r}}, \quad \mathrm{YLR}=\frac{L_{y}}{L_{y}+L_{g}+L_{r}},
$$

where $L_{g}, L_{y}$, and $L_{r}$ denote the length of green (smooth), yellow (heavy volume), and red (congested) links.

By analysis of collected behavioral data, we managed to find a set of significant factors. A binary logit model is presented in Table 2. The estimated coefficients of the variables are all significant at 95\% confidence level. The likelihood of diversion has been positively affected by certain factors, for example, age, length of routine route $l_{\mathrm{AB}}$, diversion advice, red segment ratio on the routine route $\mathrm{RLR}_{0}$ and passively affected by length of diversion route $l_{\mathrm{ACD}}$, and red segment ratio on the diversion route $R_{L} R_{1}$.

In addition, the utility function is formulated as

$$
\begin{aligned}
V_{n}(C)=V_{n}(1) & -V_{n}(0) \\
=\exp & \left(\beta_{0}+\beta_{1} \cdot \operatorname{Age}+\beta_{2} \cdot l_{\mathrm{AB}}+\beta_{3} \cdot l_{\mathrm{ACD}}\right. \\
& +\beta_{4} \cdot \mathrm{RLR}_{0}+\beta_{5} \cdot \mathrm{RLR}_{1} \\
& \left.+\beta_{6} \cdot \text { Advice }+ \text { const }\right) .
\end{aligned}
$$


TABLE 2: Binary logit model for drivers' responses to VMS.

\begin{tabular}{lcccc}
\hline Variables & Coefficient & Estimates & Standard.Error & Significance \\
\hline Age & $\beta_{1}$ & 0.044 & 0.017 & 0.010 \\
$l_{\mathrm{AB}}$ & $\beta_{2}$ & 0.965 & 0.096 & 0.001 \\
$l_{\mathrm{ACB}}$ & $\beta_{3}$ & -0.439 & 0.076 & 0.001 \\
$\mathrm{RLR}_{0}$ & $\beta_{4}$ & 3.510 & 0.606 & 0.000 \\
$\mathrm{RLR}_{1}$ & $\beta_{5}$ & -6.240 & 1.371 & 0.000 \\
Advice & $\beta_{6}$ & 0.431 & 0.211 & 0.041 \\
Constant & $\beta_{0}$ & -3.032 & 1.314 & 0.021 \\
\hline
\end{tabular}

The probability that individual $n$ chooses alternative route can be further obtained in the following equation:

$$
P_{n}(C)=\frac{V_{n}(C)}{1+V_{n}(C)} .
$$

\section{The Cooperative Mechanism of VMS and TSC}

In this section, we propose a cooperative mechanism of VMS and traffic signal control system in urban areas. In order to improve effectiveness of cooperation, a two-stage nested optimization problem is formulated.

3.1. Cooperative Mechanism. Based on the proposed logit model in Section 2, we found that the red segments ratios of an arterial displayed on VMS have a profound impact on the behavior of en route diversion decisions. Consequently, it will be a reasonable and practical choice to take the dynamic graph information as an active travel guidance tool (i.e., to display arterial with colors predicted by a reasonable and desirable method), instead of displaying current traffic status passively. With the collaboration of traffic signal control, the new VMS strategy method can not only distribute traffic flow in desirable proportions but also provide traffic status more closely to travellers' driving feeling.

This important improvement of the active VMS strategy is achieved by two preconditions: first, we allow the cooperation of VMS and TSC systems instead of isolation ones; second and more importantly, we can find a desirable solution by joint optimization in a short term and the solution has to be consistent with drivers' driving feelings for a sustainable effectiveness in a long-term use. This approach strikes a very nice balance between accessibility and effectiveness.

By considering signal cycle length and the location of VMS panels, the control period $T$ can be determined at first. As mentioned above, during the $j$ th control interval $\left(t_{j-1}, t_{j}\right)$, the cooperative strategy engenders a feasible and desired solution for the next control interval $\left(t_{j}, t_{j+1}\right)$. And the solution for $(j+1)$ th control interval, denoted as $x_{j+1}$, is a group of control variables:

$$
x_{j+1}=\left[\mathrm{RLR}_{0}, \mathrm{YLR}_{0}, \mathrm{RLR}_{k}, \mathrm{YLR}_{k}, d_{k}, C_{k}, g_{k}, \Phi_{k}\right]_{j+1},
$$

where $\mathrm{RLR}_{0}$ and $\mathrm{YLR}_{0}$ denote the red segments ratios and yellow segments ratios on the pretrip route and $\mathrm{RLR}_{k}$ and
$\mathrm{YLR}_{k}$ denote ratios on the $k$ th route; $d_{k}$ is equal to 0 or 1 and $d_{k}=1$ represents giving out the diversion suggestion for $k$ th route; $C_{k}, g_{k}$, and $\Phi_{k}$ denote the cycle times, green times, and offsets of signal controllers on the $k$ th route. We assume that there are $k$ alternative routes which can be chosen for drivers. Though the behavioral survey verifies the relation between drivers' behaviors and RLR, it is necessary to compute both RLR and YLR in the joint optimization problem because the two parameters determine the condition of an arterial road together.

When it comes to the aforesaid first precondition, both the VMS and TSC systems are installed and controlled by transportation management agencies. The implementation of the joint optimization for the two systems in the control center is easy to complete.

On the other hand, when it comes to the second precondition, some if-then rules should be satisfied for the optimization process. We assume that the control result is $y_{j+1}$ under the condition of $x_{j+1}($ see $(4))$ :

$$
x_{j+1}^{\prime}=\left[\mathrm{RLR}_{0}^{\prime}, \mathrm{YLR}_{0}^{\prime}, \mathrm{RLR}_{k}^{\prime}, \mathrm{YLR}_{k}^{\prime}\right] \text {, }
$$

where the $\mathrm{RLR}_{0}^{\prime}, \mathrm{YLR}_{0}^{\prime}, \mathrm{RLR}_{k}^{\prime}$, and $\mathrm{YLR}^{\prime}$ represent the estimation of the control results on pretrip route and other routes:

$$
\begin{aligned}
& \text { if } d_{k}=1 \\
& \text { then } \mathrm{RLR}_{k}<\mathrm{RLR}_{0}, \\
& \mathrm{YLR}_{k}<\mathrm{YLR}_{0} \\
& \mathrm{RLR}_{k}^{\prime}<\mathrm{RLR}_{k}, \\
& \qquad \mathrm{YLR}_{k}^{\prime}<\mathrm{YLR}_{k}, \\
& \text { if } d(k)=0, \quad \mathrm{RLR}_{k}<\mathrm{RLR}_{0}, \\
& \quad \mathrm{YLR}{ }_{k}<\mathrm{YLR}_{0} \\
& \text { then } \mathrm{RLR}_{k}^{\prime} \leq \mathrm{RLR}_{k}, \\
& \mathrm{YLR}_{k}^{\prime} \leq \mathrm{YLR}_{k} .
\end{aligned}
$$

Equation (6) ensures that the advice suggestions will be consistent with the graph on VMS and traffic status on actual road network. Equation (7) ensures that when there is no suggestion on panels the graph also should be consistent with traffic status on actual road network. Thus, the foremost aim of the cooperative strategy is to generate a desired and feasible traffic status on VMS panels in coordination with traffic signal control system.

3.2. The Two-Stage Nested Optimization Problem. As the mechanism mentioned above, how to find a desirable solution of VMS travel guidance and traffic signal control can be represented as an optimization problem. We name this optimization problem the VMS guidance and signal coordination (VGSC) problem. To solve this problem, traffic signal parameters (i.e., cycle times, green times, and offsets) of 
arterials shown on the VMS panels should be optimized simultaneously with these arterials' colors and diversion advice. In other words, all variables in (4) should be optimized during a control period.

Among those variables, the offsets need a special attention to their optimization. For major arterials having a number of signalized intersections, optimal offsets under fixed cycle times and green splits need to be selected from a $(n-$ 1)-dimensional solution space $C^{n-1}$ (where $C$ denotes the cycle time). As a result, it is time-consuming to optimize offsets conjunction with other parameters. For the shortage of optimizing offsets with other parameters simultaneously, a two-stage nested optimization model is formulated.

In the first stage, VMS parameters and arterial signal parameters are optimized in order to minimize the total travel time of travellers on a road network. The first-stage optimization problem can be expressed in

$$
\min F_{t}=\sum_{\forall r s \in M} \int_{0}^{T} f_{r s}(t) \eta_{r s}(t) d t,
$$

OD constraints,

$$
\begin{array}{ll}
\text { s.t. } & d_{r s}(t)=\sum_{p \in P_{r s}} f_{r s p}(t) \quad \forall r, s, t, \\
& f_{r s p}(t) \geq 0 \quad \forall r, s, t, p \in P_{r s},
\end{array}
$$

VMS constraints in (5)-(6);

Signal control constraints,

$$
C_{k} \in\left(C_{\min }, C_{\max }\right), \quad g_{k} \in\left(g_{\min }, C_{k}\right), \quad \forall k,
$$

where $f_{r s}(\mathrm{t})$ is the flow on routes between $r$ and $s ; \eta_{r s}(t)$ denotes the travel time between $r$ and $s$; $M$ denotes all $\mathrm{O}$ $\mathrm{D}$ pairs on a road network; $d_{r s}$ is the total travel demand between $r$ and $s$; $p$ represents path and $P_{r s}$ denotes all paths between $r$ and $s$. The VMS constraints have been explained above. In addition, the arterial signal optimization owns its specific constraints (see (10)).

In the secondstage, the optimization of $\Phi_{k}$ can be regarded as a nested optimization problem. The $\Phi_{k}^{\text {opt }}$ can be searched iteratively under the fixed $y_{j+1}$. The second-stage optimization problem can be expressed in

$$
\begin{aligned}
& \min G_{t}=\sum_{\forall l \in k} \eta_{l}(t) d t, \\
& \text { s.t. } \quad \Phi_{k} \in\left(0, \mathrm{C}_{k}\right),
\end{aligned}
$$

where $\eta_{l}(t)$ denotes the average travel time of link $l$ on the $k$ th path.

\section{Simulation-Based Method for the Two-stage Nested Optimization Problem}

In this section, we provide a simulation-based-optimization (SBO) method for the two-stage nested optimization problem. A SBO systematic framework is put forward in
Section 4.1. Accordingly, the optimization algorithms used in stage I and stage II are explained in detail (see Algorithms 1 and 2).

4.1. Systematic Framework. To better illustrate the cooperative mechanism, we propose a systematic framework in Figure 2. In view of the complexity of network traffic flow and interactions between different strategies, SBO methods have been introduced to solve a few engineering optimization problems, such as a decision support tool for mitigating traffic congestion [17] and regional signal timing strategies optimization [18].

With multiple decision variables in the two-stage optimization problem, the interdependence between VMS and traffic signal control is hard to evaluate by a traditional optimizer. In addition, a microscopic traffic simulator can give a relatively accurate estimation of flow distribution, average speeds, and interactions of vehicles.

Therefore, we adopt the SBO method to solve the aforementioned optimization problem. In Figure 2, the strategy module concerns operational strategies: VMS travel guidance and traffic signal control. Based on the inputs (e.g., network, demand, and strategies), the simulation module in TRNSIMS can produce detailed outputs including accurate traffic conditions and traffic flow assignment with the driver diversion module. Within the framework, the optimization module is designed to solve the first-stage optimization problem by using genetic algorithms (GAs). And the second-stage optimization problem is solved by dynamic programming method.

The SBO framework aims to obtain the feasible optimal strategies that minimize total travel time of all travellers. And the simulation-based algorithms are explained in detail as follows.

4.2. Stage I: Genetic Algorithm for Joint Optimization. As previously mentioned, the optimization of VMS and TSC is difficult to find optimal solution mathematically. As suggested in [19], biological evolution, the essence of GA, is an appealing source of inspiration for addressing computational problems. In this paper, genetic algorithm (GA) is adopted to reduce computation time. The evaluation function, control variables, constraints, and termination condition are explained as follows.

4.2.1. Evaluation Function. As indicated in (8), the objective function of the first-stage problem is the total travel time of all travellers, which can be directly obtained from the output of microsimulator module in TRANSIMS.

After simulation of each individual, the fitness function is applied to each solution indicating how close it meets the overall specification. Based on cellar automata approach, microsimulator can produce specific information for every traveller. Therefore, the temporal summary of total travel time and average speed over a segment of link can be aggregated in given time increments. It allows us to compute the objective function and constraints. 


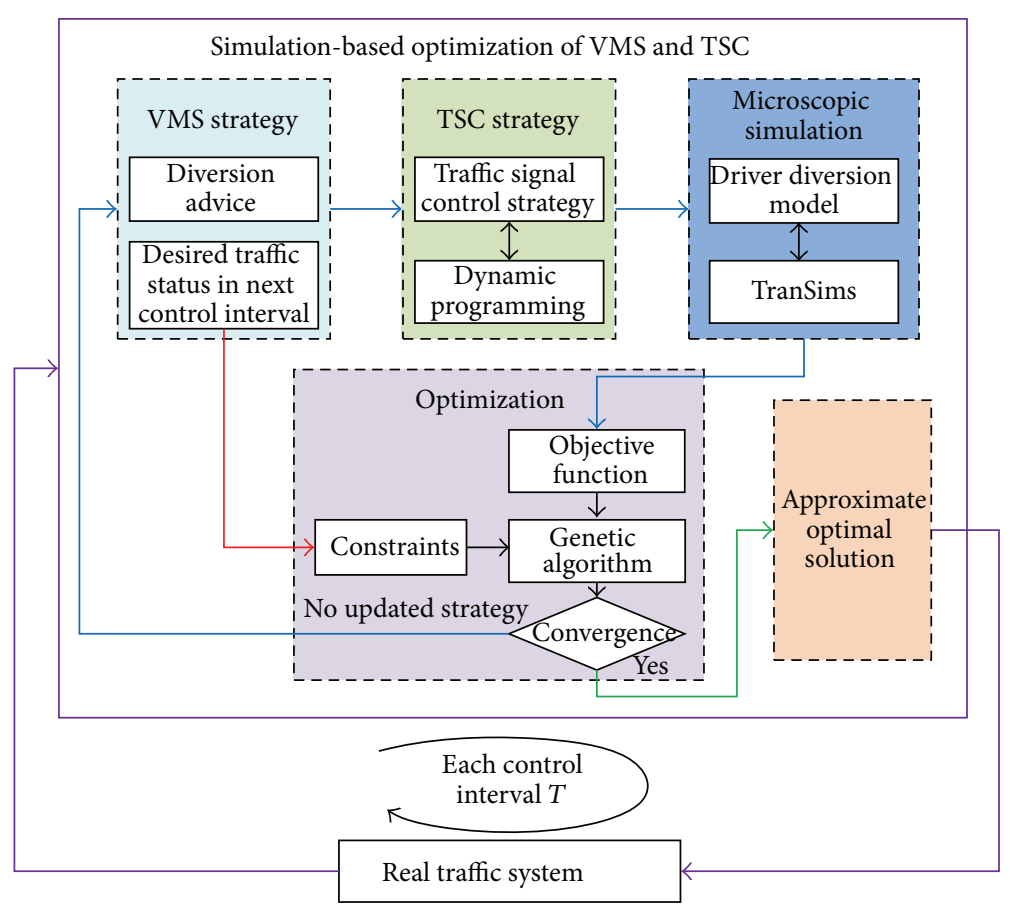

Figure 2: A SBO framework for the VGSC Problem.

Initialize $y_{j+1}=\left[\mathrm{RLR}_{0}, \mathrm{YLR}_{0}, \mathrm{RLR}_{k}, \mathrm{YLR}_{k}, d_{k}, C_{k}, g_{k}\right]_{j+1}$ as well as $\Phi_{k}=\{0\}$ (the first time) or $\boldsymbol{\Phi}_{k}^{\text {opt }}$ (optimization result in stage II).

DO the following procedure iteratively:

Find the optimal $y_{j+1}$ by solving the optimization problem;

Compute of objective function by (8) with respect to the optimal offsets

$\Phi_{k}^{\text {opt }}$ that is obtained in the second stage;

Filter solutions by constraints in (8)-(10)

Update $y_{j+1}$

Until satisfy the convergence criteria

Algorithm 1: The optimization procedures of stage I.

Initialize $y_{j+1}$ as well as $\boldsymbol{\Phi}_{k}$

DO the following procedure iteratively:

Find the optimal $\Phi_{k}$ under the condition of $y_{j+1}$;

Compute of objective function by (11)

Filter solutions by constraints in (12)

Update $\boldsymbol{\Phi}_{k}$

Until satisfy the termination criteria $\operatorname{Return} \Phi_{k}^{\text {opt }}$

Algorithm 2: The optimization procedures of stage II. 
4.2.2. Control Variables. In a genetic algorithm, a population of candidate solutions is evolved toward better solutions. Each candidate solution has a set of variables (i.e., VMS parameters: $\mathrm{RLR}_{0}, \mathrm{YLR}_{0}, \mathrm{RLR}_{k}$, and $\mathrm{YLR}_{k}, d_{k}$; signal parameters: $C_{k}$ and $g_{k}$ ).

4.2.3. Constraints. Because of the nature of genetic algorithms, wrapping or truncating individuals in a generation has great influence on optimization performance. In order to deal with the constraints mentioned above without noises, we take advantage of a penalty scheme to the evaluation function. It provides a penalty to the fitness, which is proportional to the constraint violation.

4.2.4. Termination Condition. The iterative process is repeated until a termination condition has been reached. The terminating conditions are as follows.

(i) Max generation: reach the max generation.

(ii) Convergence criteria: reach a plateau that successive iterations no longer produce better results.

4.3. Stage II: Dynamic Programming for Offsets Optimization. The second-stage optimization problem was the arterial signal offsets optimization under the condition of fixed green time and cycle time. A recent research by Gartner and Rahul developed a dynamic programming (DP) model which is suitable for signal offsets optimization [20]. On this basis, we further integrate this DP model with simulations in TRANSIMS. In detail, the basic procedure of DP model in a five-intersection arterial is described as follows.

The arterial signalized intersection is denoted as node $=$ $0,1,2,3,4$. Correspondingly, the arterial links are defined as link $=1,2,3,4$. For simplicity, we do not show the branches in Figure 3 while taking into account traffic flows of branches when simulating in TRANSIMS. The set of offsets for different nodes is defined as $\Phi=\left[\Phi_{1}, \Phi_{2}, \Phi_{3}, \Phi_{4}\right]$.

Initialization. The DP model needs original input parameters including cycle time $(C)$ and green time $(G)$ and other basic inputs for simulations.

Dynamic Programming. A process of DP is illustrated in Figure 4.

(1) By setting offset interval $\delta_{\text {offset }}$, there are $N_{j}$ offsets for node $j$ and $N_{j}$ can be determined by $N_{j}=C_{j} / \delta_{\text {offset }}$.

(2) Every connection in Figure 4 means the average travel time on links. For link $j(j=2,3$, and 4$)$, offset $\Phi_{j}$ between nodes $j$ and $j-1$ will be associated with former offset sequence:

$$
\Phi_{j-1}=\left[\Phi_{1}, \ldots, \Phi_{j-1}\right] .
$$

(3) The average travel time on link $j$ can be further computed by TRANSIMS, which can be denoted as $\mathrm{TT}_{j}$.
(4) By comparing this average travel time, we could obtain the offset $\Phi_{j}$ and get a temporary optimized offset sequence from $\Phi_{1}$ to $\Phi_{j}$.

(5) By repeating the above 3 steps for each link, the optimal offset sequence $\Phi_{\text {opt }}^{C, g}$ can be finally determined under the given cycle time and green time.

(6) A sum of average travel times for the set of links in the arterial is a significant parameter for evaluating the arterial signal control effectiveness. Corresponding to the optimal offset sequence $\Phi_{\mathrm{opt}}^{\mathrm{C}, \mathrm{g}}$, the minimum travel time can be obtained by microsimulator in TRANSIMS:

$$
\mathrm{TT}_{\min }^{C, g}=f\left(\Phi_{1}, \ldots, \Phi_{i}\right)
$$

\section{Application of Methodology}

5.1. Network Topology. The proposed methodology is applied in the actual road network of Tianjin Binhai Hi-Tech Industrial Development Park (T.H.I.P, China). This site is located $3 \mathrm{~km}$ west to Tianjin urban area, with a two-square-kilometer core area. As a connection to the Tianjin municipal area, road network in this core area is almost filled with heavy traffic flow during rush hours of working days.

In this area, more than 80 percent traffic is due to daily commuters, who work in T.H.I.P. Moreover, traffic management rules and traffic signals are properly maintained, while daily traffic congestions still trouble travelers. Therefore, the road network of T.H.I.P is selected for the proposed study (as shown in Figure 5). It has 30 nodes and 46 unidirectional links. Among them, there are two arterials, Fukang Road (Path 1, $1.97 \mathrm{~km}$, in Figure 6) and Yingshui Road (Path 2, $3.9 \mathrm{~km}$, in Figure 6), which carry the most traffic volume from the Tianjin urban area to T.H.I.P during morning peak hours which remain from 8:00 to 10:00. Besides the links and nodes inside this area, we also set some external zones (blue trapezoids in Figure 6) on the boundary of the road network which are connected with external links to the major traffic flow.

Subsequently, the study site network was coded into TRANSIMS manually by using GIS networks. The network creation required static information including zone, node, link, pocket lane, and vehicle composition. Among them, the vehicle composition contains vehicle type, size, capacity, maximum speed, and acceleration. The traffic signal timing plan for arterial signal control consists of cycle time, green time, yellow time, red time, offset, and phase sequence. And the timing plan can be revised by signal control module dynamically.

If the initial simulation result indicates that default parameters of microsimulator module in TRANSIMS are not acceptable, the parameter calibrations are necessary. In this paper, calibrations for the AM and PM peak time periods were conducted. And the urban arterial network mentioned above was calibrated against field measured traffic count data and travel times by video cameras at signalized intersections. 


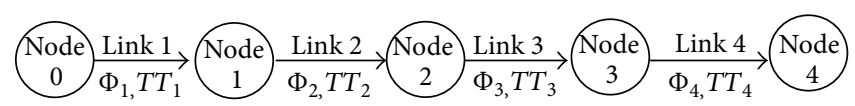

FIGURE 3: Offsets and travel time functions on a five-intersection arterial.

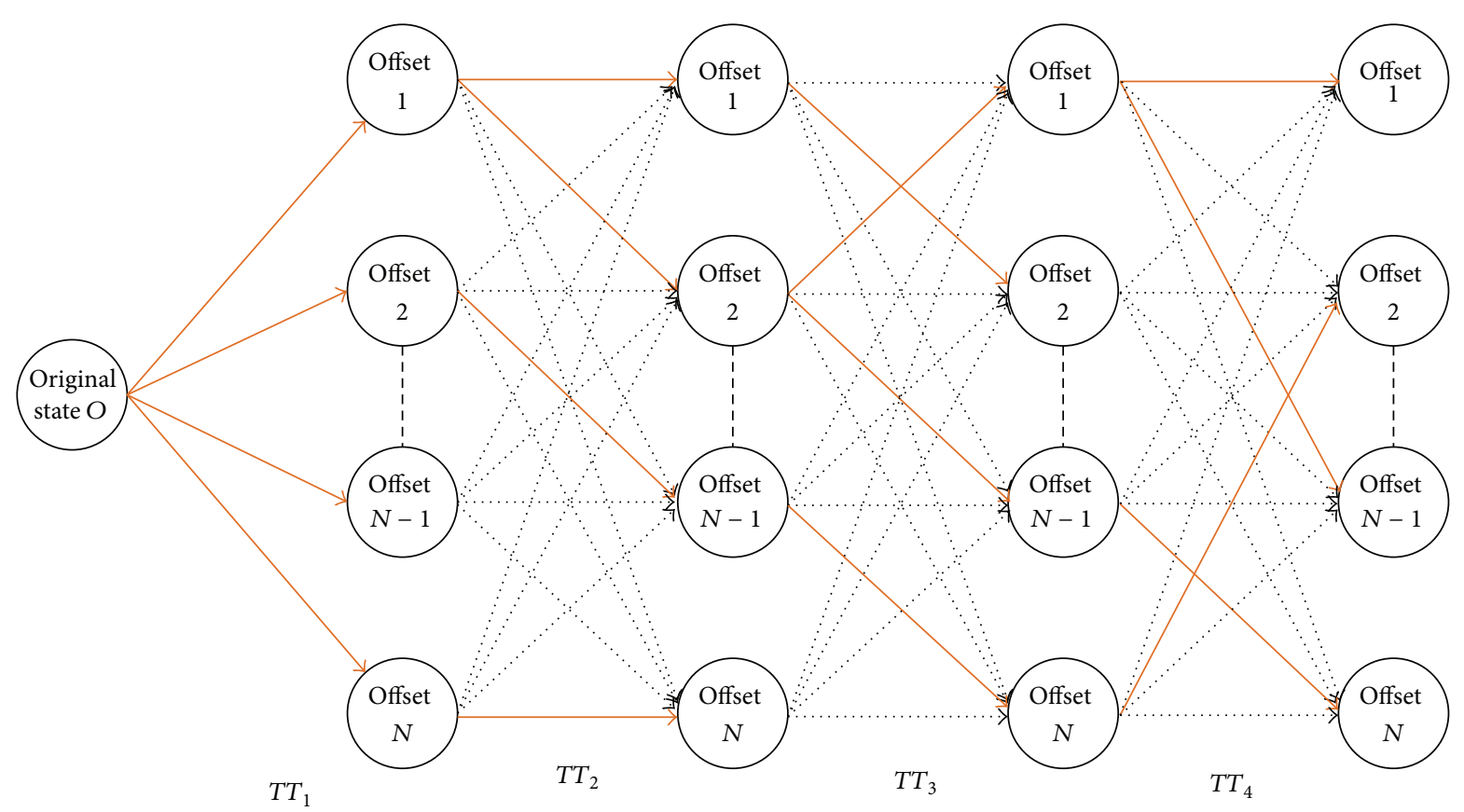

FiguRE 4: An illustration of dynamic programming of offsets.

Based on the basic steps of parameter calibration in TRANSIMS proposed by Park and Kwak [21], we introduce the calibration procedures in detail as follows.

5.1.1. Selection of Calibration Parameters. The first step is the selection of calibration parameters including lane-changing and car-following parameters. In brief, we take the following 5 key calibration parameters as examples.

Maximum Speed. The maximum speed is defined as the $10 \mathrm{~km} / \mathrm{h}$ above the limited speed.

Slow-Down Probability. The slow-down percentage defines the likelihood that a vehicle will slow down for no apparent reason. The default value is zero (i.e., no random slow-down).

Slow-Down Percentage. The slow-down percentage defines the amount a vehicle will randomly slow down. The default value is zero.

Maximum Waiting Time. The maximum waiting time defines when a vehicle is removed from the simulation. If the vehicle has not moved for this amount of time, the vehicle is removed from the link and moved to the destination parking lot. The default value is 60 minutes.

Maximum Swapping Speed. To avoid deadlock situations, a cooperative lane swapping concept permits the vehicles to continue their trips. This parameter defines the maximum speed at which lane swapping will be allowed. The default value is $37.5 \mathrm{~m} / \mathrm{s}$.

5.1.2. Experimental Design and Multiple Runs. Subsequently, ranges of calibration parameters are determined and multiple sets of calibration parameters are generated. In detail, slowdown probability ranges from 0 to $50 \%$; slow-down percentage ranges from 0 to 50\%; maximum waiting time ranges from 60 to 200 minutes; and maximum swapping speed ranges from 0 to $30 \mathrm{~m} / \mathrm{s}$. And then 1000 simulation runs are performed for each parameter set.

5.1.3. Feasibility Test. The distribution of simulation results is compared to travel times and volumes generated by field video cameras in order to determine whether current parameter ranges are feasible. As a result, the parameters are calibrated and the results are that the slow-down probability 


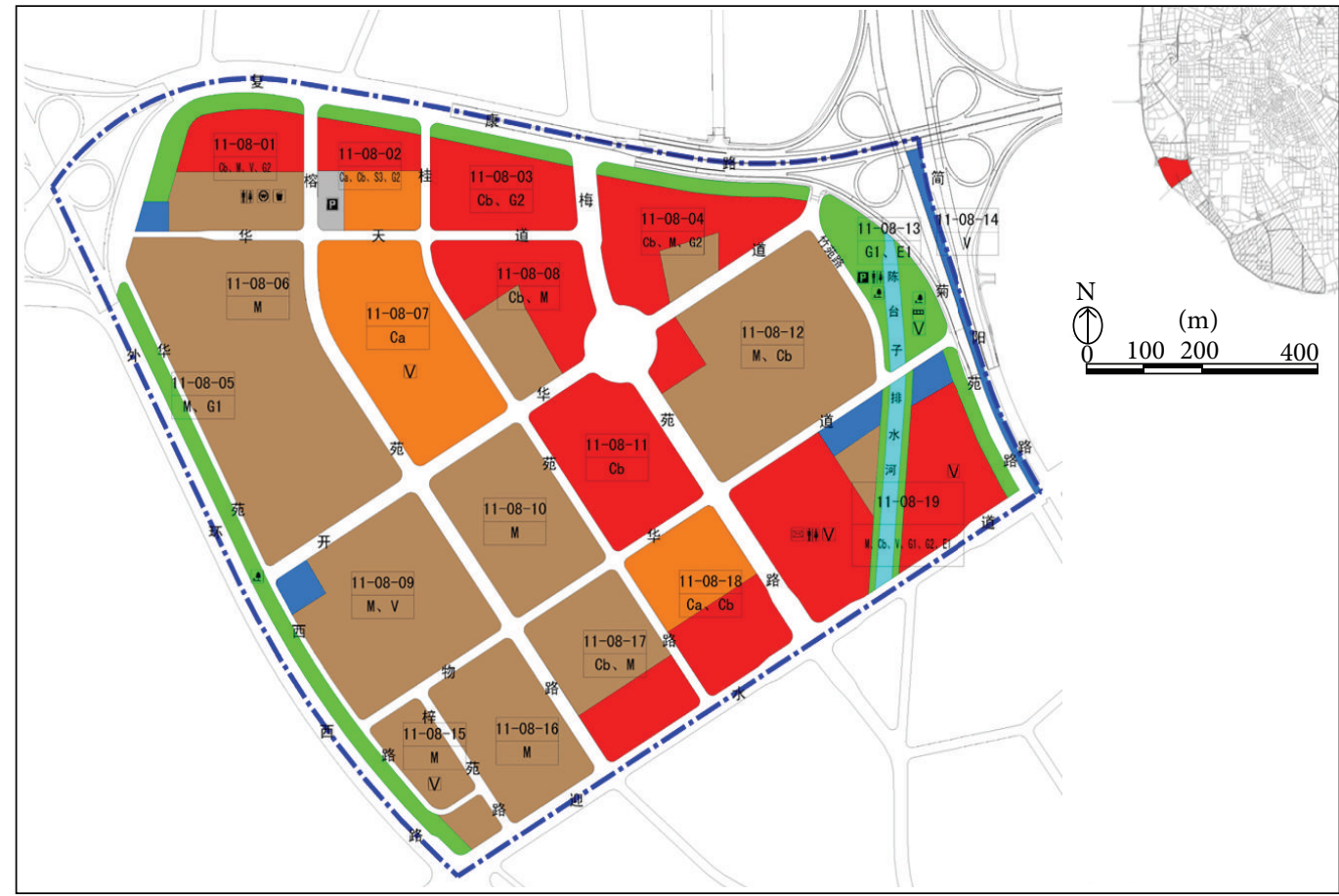

Residential land

Commercial and financial land

Public welfare land

Industrial land

Storage land

Public vehicle parking land

Public transportation land
Public land for public facilities

Public green land

Water

$x x-x-x$ Land property code

Border line of city roads

Track line

FIgURE 5: Map of T.H.I.P with land use.

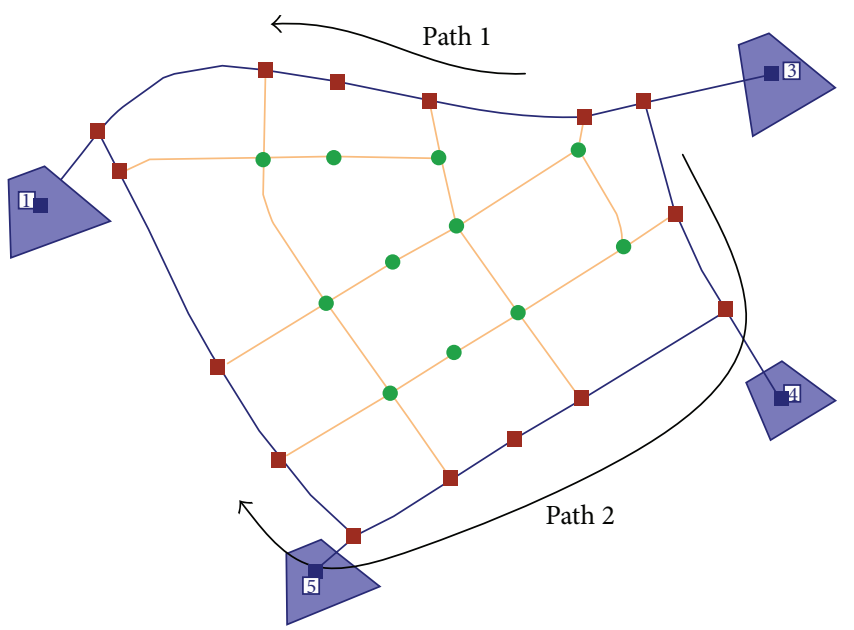

FIGURE 6: Road network topology of T.H.I.P.

is $25.3 \%$, slow-down percentage is $34.5 \%$, maximum waiting time is 83 minutes, and maximum swapping speed is $11.5 \mathrm{~m} / \mathrm{s}$.
5.1.4. Evaluation of Calibrated Parameters. In the end, it is necessary to conduct 1000 simulation runs to consider the variability of the parameter set.

5.2. Simulation-Based Optimization Process on TRANSIMS. TRANSIMS is a travel demand modeling software package that was initially developed by the Los Alamos National Laboratory (LANL) [22]. TRANSIMS separates the simulation process into two stages.

Traffic Assignment. The router module performs traffic assignment using a time-dependent minimum impedance path algorithm based on travel time of links.

Microscopic Simulation. Load travel plans, from the router module, and further computes the time-space information of vehicles second by second.

The open-source software package allows the user to develop new function and customize features of the simulation model. In order to optimize the joint strategy, we develop the driver diversion module, traffic signal control module, 


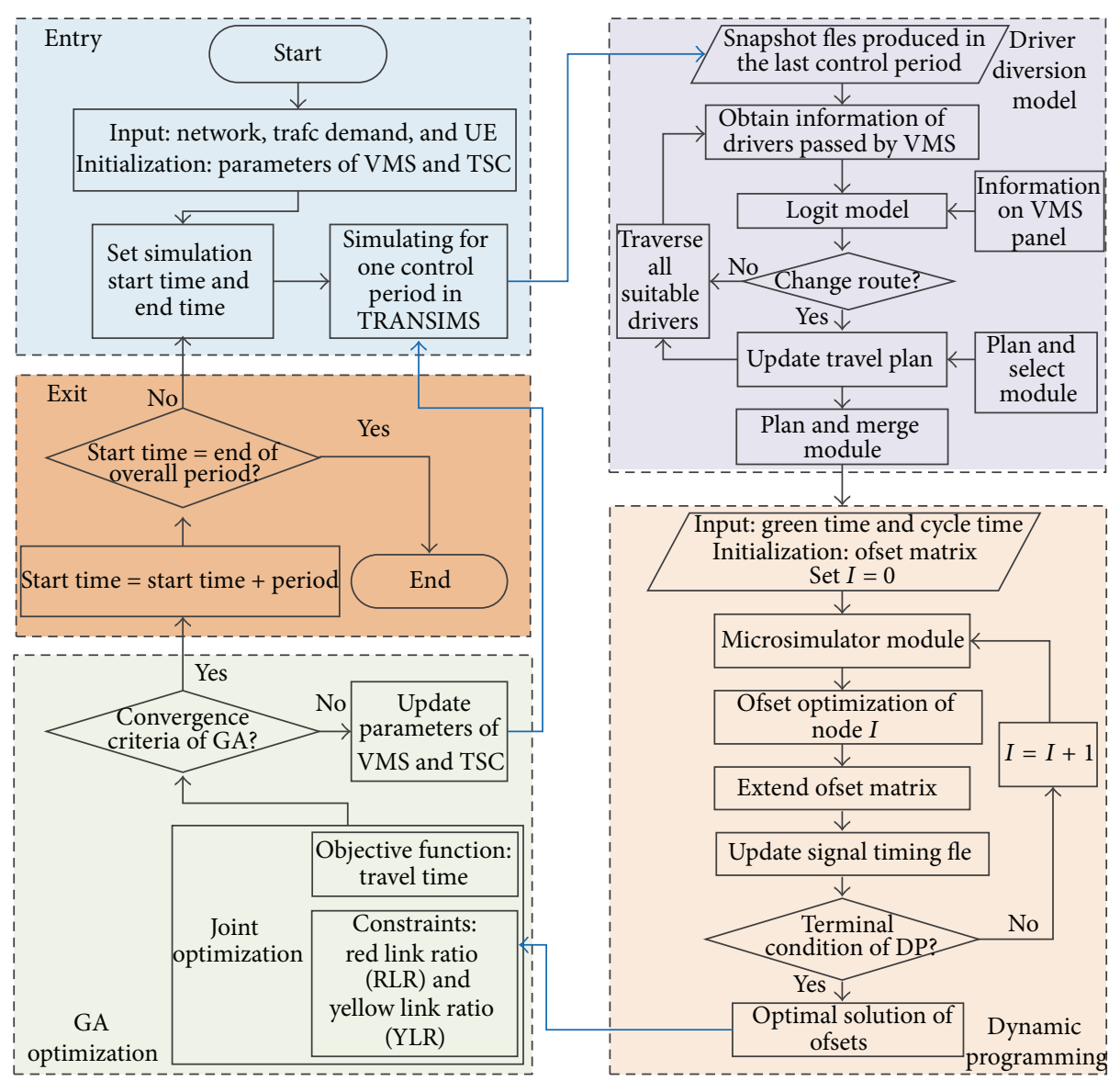

FIGURE 7: Flowchart of simulation program in TRANSIMS.

and optimization module in Python 2.7. Through integrating with postprocessor of TRANSIMS written in $\mathrm{C}++$, the developed three modules can perform various functions. A flowchart in Figure 7 shows the developed procedure in TRANSIMS to solve the joint optimization problem.

At first, the driver diversion module extracts driver spacetime information from snapshots files to find drivers who can see the VMS panels. Subsequently, the driver diversion model determines if drivers will make a detour under the influence of VMS information, personal features, and other factors identified previously.

Subsequently, the traffic signal control module can revise signal timing table under the direction of dynamic programming. And total average travel time on arterial links provides a feedback to the signal controllers to evaluate its effectiveness.

The optimization module is developed to evaluate the joint optimization by using genetic algorithm. And the statistical analysis of the simulation results was done to validate the feasibility of solutions and compute the objective value of solutions. The algorithm was implemented in Python using the Pyevolve module [23] and Networkx module [24]. In addition, parallel implementation of the GA is carried out in the optimization process in order to improve the computation effectiveness. Because of the independence of individuals in a generation, the optimization module can submit population to job manager and evaluate the simulation results after all individuals have completed simulations.

In this network, all 30 nodes are signalized nodes. Among them, 15 nodes on the two arterials (i.e., path 1 and path 2) can be controlled under the direction of optimization module, and other intersections used the fixed timing table. By using the arterial signal control method, we assume that the cycle time and green time for intersections on an arterial are the same. To make faster computation, we further restrict the range of variables' values, as follows.

(1) Cycle times: minimum cycle time $C_{\min }$ is assumed to be $80 \mathrm{~s}$ and maximum cycle time $C_{\max }$ is assumed to be $130 \mathrm{~s}$.

(2) Phase sequence: phase sequence of a given node is kept the same.

(3) Green time: minimum green time $g_{\min }$ is assumed to be $20 \mathrm{~s}$ and the maximum green time is assumed to be $C-g_{\min }$.

(4) Offsets: minimum green time $\Phi_{\min }$ is assumed to be $0 \mathrm{~s}$ and the maximum green time is assumed to be $C$. 


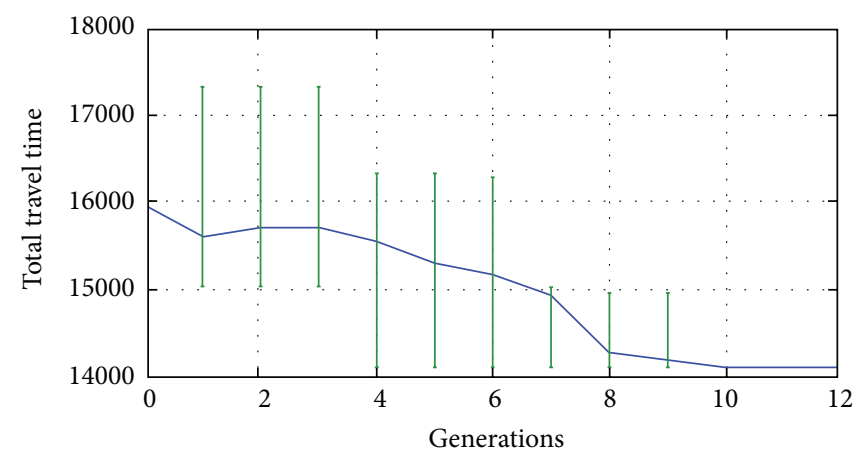

(a) The evolution process

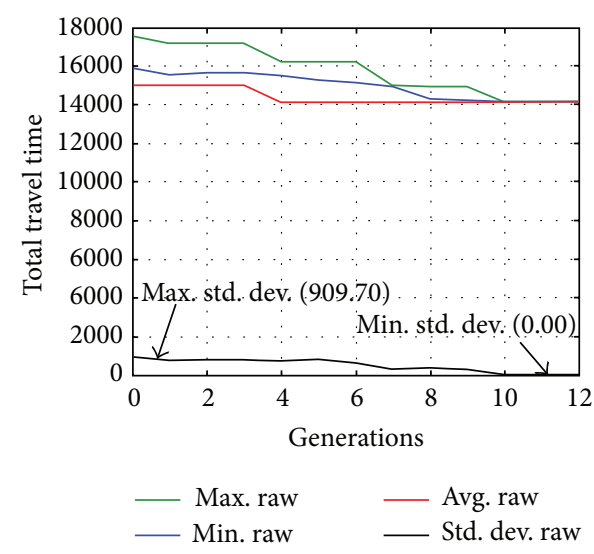

(b) The standard deviation of population in generations

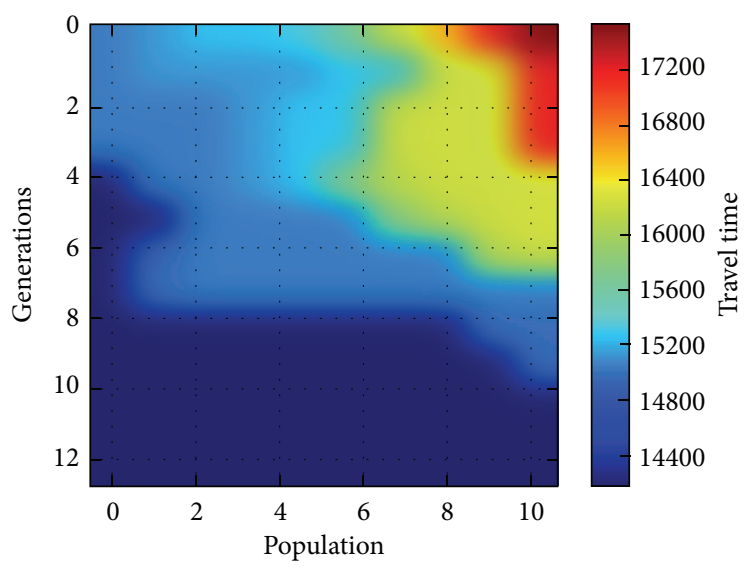

(c) Total travel time of population along generations

FIGURE 8: Convergence process of the genetic algorithm.

5.3. Simulation Results and Analysis. According to the size of study network, we set the control interval as 5 minutes. And the 12 optimum solutions can be obtained during 8:00-9:00 in the morning peak time. During each interval, 7 variables are optimized in the first-stage GA optimization, including the desired yellow ratios on the two arterials, the cycle times and green times for each arterial, and diversion advice. Moreover the offsets of 10 signalized intersections on the two arterials are optimized by the second-stage dynamic programing.
By the multiruns of the three control methods under traffic demand during the morning peak time, we present the mean values of 20 groups. The comparison of improvement of traffic condition by different strategies is shown in Table 3. The improvement by adopting the active VMS strategy with fixed signal control is $10.2 \%$, while the cooperation of both strategies reaches $26.3 \%$.

The convergence process of each control interval is further explained in Figure 8. Obviously, there exists an obvious 
TABLE 3: Comparison of minimum values of objective function.

\begin{tabular}{lcc}
\hline Control method & \multicolumn{1}{c}{ Index } \\
& $\begin{array}{c}\text { Objective function } \\
\text { values }\end{array}$ & Improvement \\
\hline $\begin{array}{l}\text { Pretimed signal control } \\
\text { without VMS }\end{array}$ & 84756.77 & $/$ \\
$\begin{array}{l}\text { Pretimed signal control } \\
\text { with VMS strategy }\end{array}$ & 76118.13 & $10.2 \%$ \\
$\begin{array}{l}\text { The cooperative strategy } \\
\text { of VMS and arterial } \\
\text { signal control }\end{array}$ & 62391.83 & $26.3 \%$ \\
\hline
\end{tabular}

trend of convergence with the generation increase as shown in Figure 8(a). The maximum number of generations is set to 20 ; the mutation rate is 0.02 ; and the crossover rate is 0.9 . When the optimization comes to the 10th generation, the standard deviation is close to 0 , which satisfies the convergence criteria in GA in Figure 8(b). It can be explicitly displayed that the objective function values of each individual from the 10th generation are kept the same as shown in Figure 8(c).

\section{Conclusion and Future Work}

In this paper, we study variable message sign (VMS) widely deployed on major arterials in developing countries. In order to appropriately describe drivers' response to VMS, we propose an en route diversion model based on a statedpreference behavioral survey. The vital significance of this new model lies in well considering drivers' responses to graphical road with colors.

On this basis, we propose a cooperative mechanism of VMS travel guidance and arterial signal control for urban traffic management. In this mechanism, traffic control parameters and VMS parameters are optimized together. Particularly for arterial signal control, a two-stage nested optimization problem is formulated. To find the optimal solution, we apply a simulation-based optimization framework for solving this two-stage nested optimization problem. In this cooperative strategy, we allow the communication between VMS and TSC systems to find a desirable and feasible solution during each control interval.

As shown in Table 3, the cooperation strategy managed to reduce total travel time of all travellers when compared to pretimed signal control without VMS strategy and pretimed signal parameters with VMS strategy. Moreover, we examine the convergence process of each interval in Figure 8. It shows that this simulation-based method strikes a very nice balance between accessibility and effectiveness. And the convergence performance is quick and effective.

Besides, it should be pointed out that this simulationbased-optimization method requires relatively high computation source by utilizing microscopic traffic simulation. For future works, we will focus on testing the proposed method for more complex networks of intersecting arterials to investigate effectiveness of the cooperative strategy. In addition, analytical methods will be researched in this cooperative mechanism to reduce computation costs.

\section{Conflict of Interests}

The authors declare that there is no conflict of interests regarding the publication of this paper.

\section{Acknowledgments}

This work was supported in part by Hi-Tech Research and Development Program of China under Grant 2014BAG03B04, National Natural Science Foundation of China Grant 51138003, and National Science and Technology Major Project 2012ZX03005016002.

\section{References}

[1] M. Papageorgiou, C. Diakaki, V. Dinopoulou, A. Kotsialos, and Y. Wang, "Review of road traffic control strategies," Proceedings of the IEEE, vol. 91, no. 12, pp. 2043-2065, 2003.

[2] N. B. Hounsell, B. P. Shrestha, J. Piao, and M. McDonald, "Review of urban traffic management and the impacts of new vehicle technologies," IET Intelligent Transport Systems, vol. 3, no. 4, pp. 419-428, 2009.

[3] A. C. Sutandi, "Evaluation of the impacts of VMS on traffic performance measures in an urban area in Indonesia," Civil Engineering Dimension, vol. 10, no. 1, pp. 28-34, 2008.

[4] S. Peeta, J. L. Ramos, and R. Pasupathy, "Content of variable message signs and on-line driver behavior," Transportation Research Record, vol. 1725, no. 1, pp. 102-108, 2000.

[5] P. Mirchandani and L. Head, "A real-time traffic signal control system: architecture, algorithms, and analysis," Transportation Research C: Emerging Technologies, vol. 9, no. 6, pp. 415-432, 2001.

[6] R. E. Allsop, "Some possibilities for using traffic control to influence trip distribution and route choice," Transportation and Traffic Theory: Proceedings, vol. 6, pp. 345-373, 1974.

[7] M. J. Smith, "The existence, uniqueness and stability of traffic equilibria," Transportation Research B, vol. 13, no. 4, pp. 295304, 1979.

[8] M. J. Smith, "The existence and calculation of traffic equilibria," Transportation Research B, vol. 17, no. 4, pp. 291-303, 1983.

[9] M. O. Ghali and M. J. Smith, "A model for the dynamic system optimum traffic assignment problem," Transportation Research B, vol. 29, no. 3, pp. 155-170, 1995.

[10] N. H. Gartner and C. Stamatiadis, "Integration of dynamic traffic assignment with real-time traffic adaptive control system," Transportation Research Record, vol. 1644, no. 1, pp. 150-156, 1998.

[11] A. S. Abdelfatah and H. S. Mahmassani, "System optimal time-dependent path assignment and signal timing in traffic network," Transportation Research Record, vol. 1645, no. 1, pp. 185-193, 1998.

[12] S. Lu, X. Liu, and Z. Yang, "Study on consistent and integrated traffic management model," Journal of Wuhan University of Technology, Transportation science and Engineering, vol. 32, no. 2, pp. 251-254, 2008 (Chinese).

[13] H. Cui, The Coordination Strategy Between Signal Control and VMS and Optimization for information Publishing, Beijing Jiaotong University, Beijing, China, 2011 (Chinese). 
[14] C. Lin, B. Gong, D. Zhao, and X. Liu, "Traffic control and VMS collaborative technique in sudden disaster," Journal of Traffic and Transportation Engineering, vol. 12, no. 6, pp. 104-110, 2012 (Chinese).

[15] J. J. Cheng and P. E. Firmin, "Perception of VMS effectiveness: a British and Canadian perspective," in Proceedings of the 12th International Conference on Road Transport Information and Control (RTIC '04), pp. 175-185, London, UK, April 2004.

[16] M. Kraan, N. van der Zijpp, and B. Tuter, "Evaluating network wide effects of VMS's in the Netherlands," in Proceedings of the 78th Annual Meeting of the Transportation Research Board, Washington, DC, USA, 1999.

[17] S. H. Melouk, B. B. Keskin, C. Armbrester, and M. Anderson, "A simulation optimization-based decision support tool for mitigating traffic congestion," Journal of the Operational Research Society, vol. 62, no. 11, pp. 1971-1982, 2011.

[18] C. Osorio, Mitigating network congestion: analytical models, optimization methods and their applications [Ph.D. dissertation], École Polytechnique Fédérale de Lausanne, Lausanne, Switzerland, 2010.

[19] M. Melanie, An Introduction to Genetic Algorithms, 3, MIT Press, Cambridge, Mass, USA, 5th edition, 1999.

[20] N. H. Gartner and D. Rahul, "Dynamic programming approach for arterial signal optimization," in Proceedings of the 92nd Annual Meeting of the Transportation Research Board, No. 130177, 2013.

[21] B. B. Park and J. Kwak, "Calibration and validation of TRANSIMS microsimulator for an urban arterial network," KSCE Journal of Civil Engineering, vol. 15, no. 6, pp. 1091-1100, 2011.

[22] L. Smith, R. Beckman, and K. Baggerly, "TRANSIMS: transportation analysis and simulation system," Tech. Rep. LA-UR95-1641, Los Alamos National Laboratory (LANL), Los Alamos, NM, USA, 1995.

[23] C. S. Perone, "Pyevolve: a Python open-source framework for genetic algorithms," ACM SIGEVOlution, vol. 4, no. 1, pp. 1220, 2009.

[24] A. Hagberg, P. Swart, and D. S. Chult, "Exploring network structure, dynamics, and function using NetworkX," Tech. Rep. LA-UR-08-05495, LA-UR-08-5495, Los Alamos National Laboratory (LANL), Los Alamos, NM, USA, 2008. 


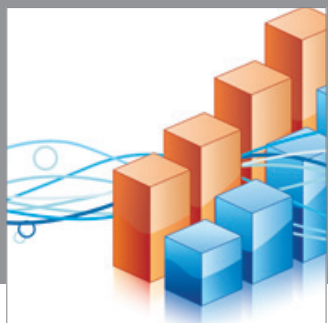

Advances in

Operations Research

mansans

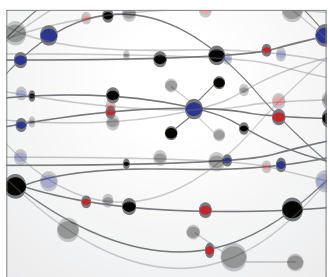

The Scientific World Journal
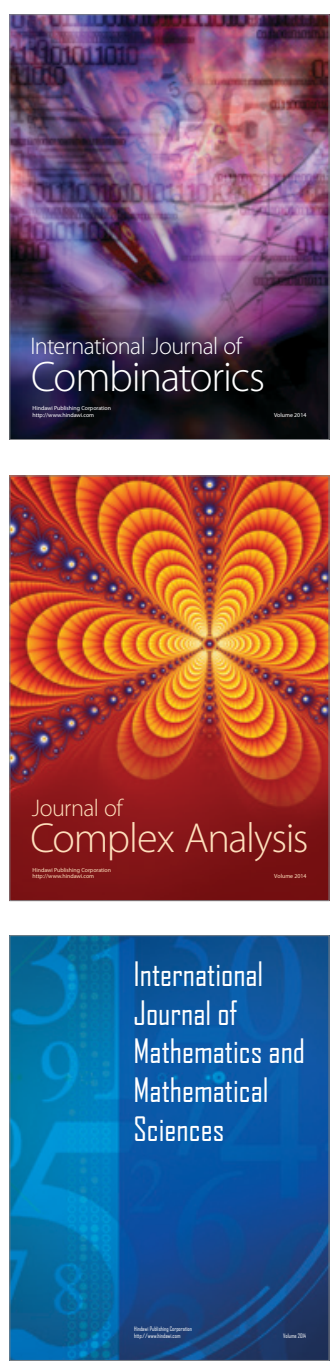
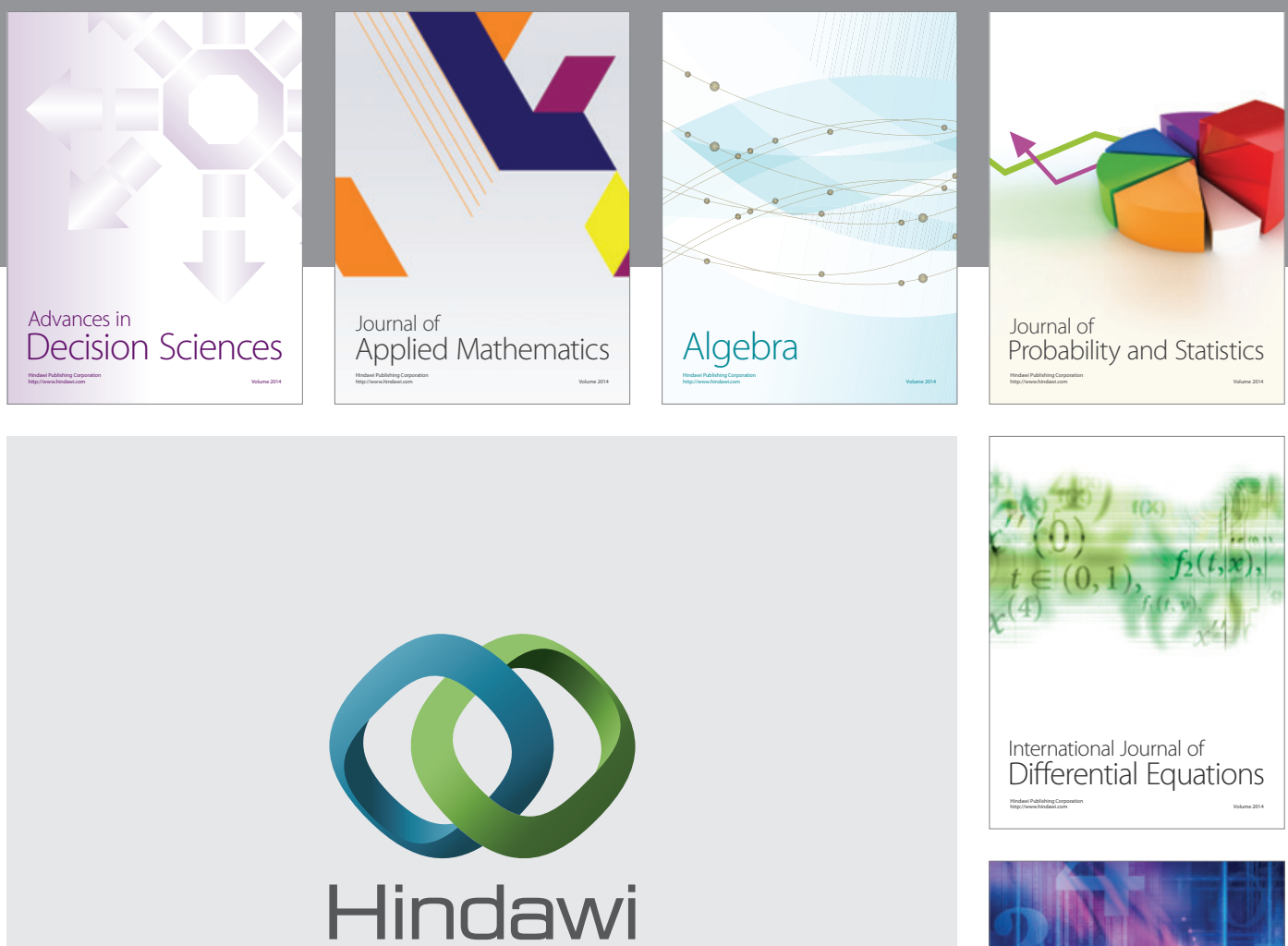

Submit your manuscripts at http://www.hindawi.com
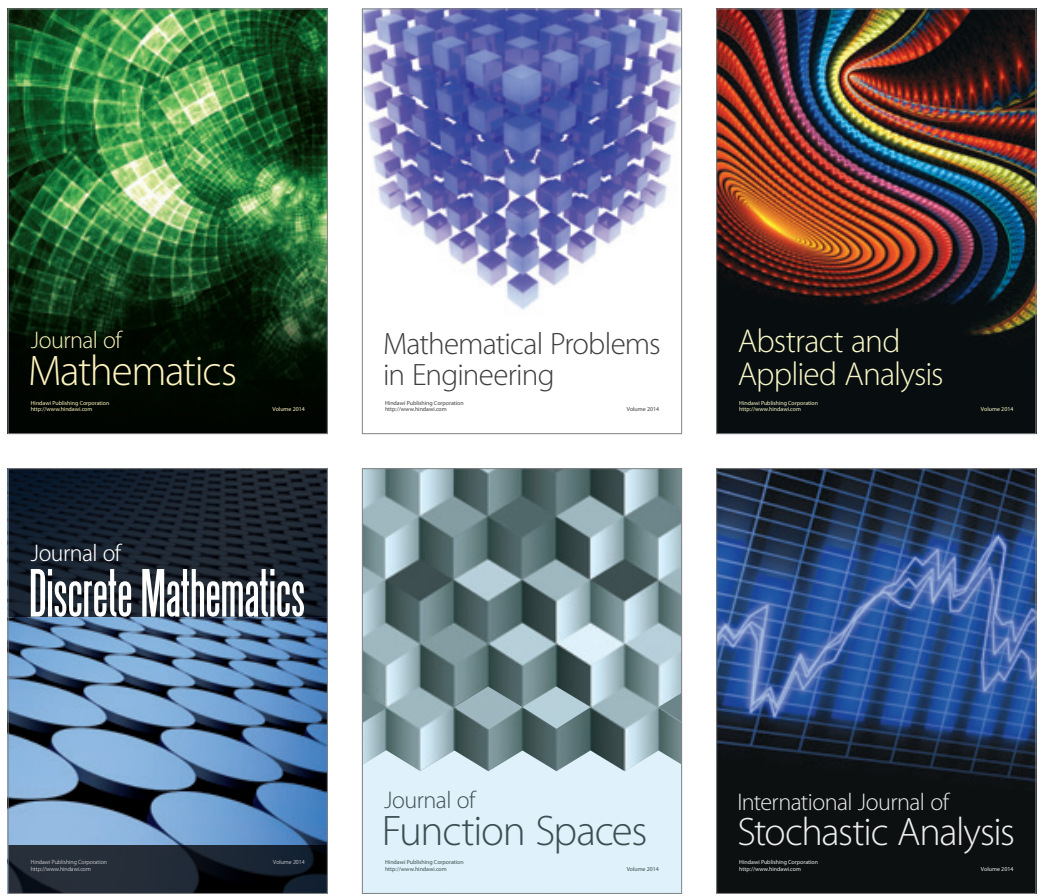

Journal of

Function Spaces

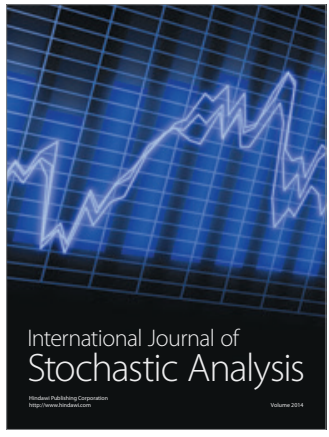

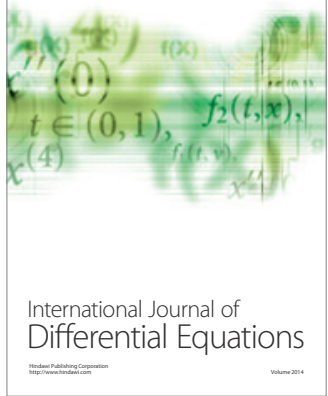
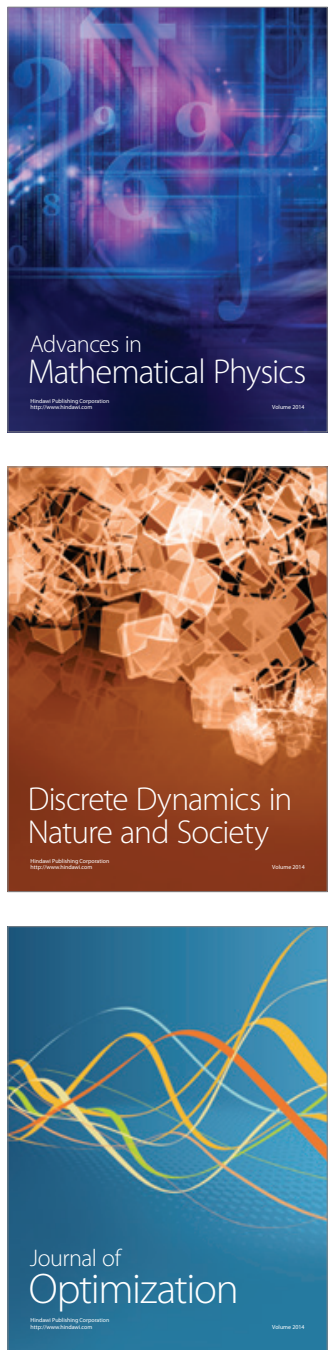